\title{
Cerebral Venous Thrombosis: A Path Less Travelled and making a Difference
}

\author{
Joy Vijayan ${ }^{1}$ and Vijay K Sharma ${ }^{1,2^{*}}$ \\ ${ }^{1}$ Division of Neurology, National University Hospital, Singapore \\ ${ }^{2}$ Yong Loo Lin School of Medicine, National University of Singapore, Singapore
}

*Corresponding author: Dr. Vijay K Sharma, Division of Neurology, Department of Medicine, National University Hospital, 5 Lower Kent Ridge Road, Singapore 119074, Tel: 65- 67724126; Fax: 65-68723566; E-mail: drvijay@singnet.com.sg

Received date: June 23, 2014, Accepted date: June 26, 2014, Published date: June 30, 2014

Copyright: (c) 2014 Vijayan J, et al. This is an open-access article distributed under the terms of the Creative Commons Attribution License, which permits unrestricted use, distribution, and reproduction in any medium, provided the original author and source are credited.

Keywords: Venous sinus thrombosis; Cerebral venous infarction; Anticoagulation

\section{Cerebral Venous Thrombosis: A Path Less Travelled and Making a Difference}

Thrombosis of the cortical veins or the cerebral venous sinuses is not an uncommon cause of stroke, especially in the young and those with underlying medical co-morbidities like malignancy or systemic connective tissue disorders [1,2]. Unlike arterial events that present suddenly, venous thrombosis can present in either an indolent manner with evolving headache, visual impairment or, more commonly, in a catastrophic manner with seizures, altered mentation or focal neurological deficits.

Clinical manifestations of cerebral venous thrombosis (CVT) largely depend on the location of the thrombosis. The superior sagittal sinus is most commonly involved and often presents with headache, increased intracranial pressure, and papilledema [3]. Early diagnosis and initiation of treatment is important to maximize the probability of a favorable outcome. This would entail adopting a three-pronged approach which would include: 1) curtailing the extension of the thrombotic process, 2) identifying and managing associated complications like seizures, intracranial hemorrhage and raised intracranial pressure and 3) identifying and treating the underlying cause of thrombosis.

The diagnosis of thrombosis of the cortical veins or the dural venous sinuses is based on imaging studies that include computed tomography (CT) or magnetic resonance (MR) of the brain. CT or MR venography can directly assess the venous system and confirm the diagnosis. In addition, the extent of thrombosis may also be determined for planning management strategies and better prognostication $[4,5]$.

Although thrombophilia work up is rewarding only in about a quarter of the patients with CVT, all efforts should still be made to identify the causative mechanism. It is important to screening for connective tissue disorders, malignancy and parameningeal source of infection in addition to the thrombophilic states [6].

The mainstay of treatment is prevention of propagation of the thrombotic process. Guidelines issued by the American Heart Association-American Stroke Association, supported by two randomized trials and several observational studies, have highlighted the usefulness of anticoagulant therapy in achieving a favorable outcome [7]. Patients started on anticoagulants had reduced occurrence of headache, seizures, focal deficits and intracranial hemorrhage [6,7]. There are no data supporting the choice of dose adjusted unfractionated heparin over low molecular weight heparin with regards to outcome, although some recent reports suggest that the latter may be associated with reduced risk of hemorrhage and thrombotic complications in a subset of patients with deep venous thrombosis or pulmonary embolism. The role of anticoagulation in achieving a better outcome in patients with pre-existing intracranial hemorrhage has also been demonstrated [7]. Thus, early anticoagulation should be instituted in all patients with CVT regardless of the presence of intracranial hemorrhage. The duration of anticoagulation the survivors remains debatable.

Treatment beyond anticoagulants needs to be considered in some patients who deteriorate clinically or do not respond in an expected manner [8]. The options include direct catheter thrombolysis or mechanical thrombectomy. Recent studies have shown fibrinolytics to reduce mortality in critically ill patients, albeit with an increased risk of intracranial hemorrhage. Patients who fail to respond to direct catheter thrombolysis can be considered for mechanical thrombectomy, either by Balloon-Assisted thrombectomy followed by thrombolysis or by clot retrieval using the Merci retrieval device or the penumbra system [9].

The management of venous thrombosis is not complete without concurrently treating for seizures if they arise and assessing for symptoms and signs of raised intracranial pressure [6]. The threshold to initiate antiepileptic medications should be very low and may require the use of more than one anti-epileptic medications are times. Life-threatening intracranial hypertension may require the need for decompressive craniectomy in some cases [10].

Finally, despite the lack of solid traditional scientific evidence, anticoagulation remains the simplest management option [11]. However, the management can be complex in a considerable proportion of CVT patients requiring a multi-disciplinary approach, largely due to increasing intracranial pressure. The ongoing innovative research is expected to help in developing more effective therapeutic strategies to ensure better outcomes for patients with CVT.

\section{References}

1. Stam J (2005) Thrombosis of the cerebral veins and sinuses. N Engl J Med 352: 1791-1798.

2. Stam J (2003) Cerebral venous and sinus thrombosis: incidence and causes. Adv Neurol 92: 225-232.

3. Biousse V, Ameri A, Bousser MG (1999) Isolated intracranial hypertension as the only sign of cerebral venous thrombosis. Neurology 53: 1537-1542.

4. Leach JL, Fortuna RB, Jones BV, Gaskill-Shipley MF. Imaging of cerebral venous thrombosis: current techniques, spectrum of findings, and diagnostic pitfalls. Radiographics 26: S19-S41.

5. Poon CS, Chang JK, Swarnkar A, Johnson MH, Wasenko J (2007) Radiologic diagnosis of cerebral venous thrombosis: pictorial review. AJR Am J Roentgenol 189: S64-75.

6. Ferro JM, Canhão P, Stam J, Bousser MG, Barinagarrementeria F; ISCVT Investigators (2004) Prognosis of cerebral vein and dural sinus thrombosis: 
Citation: Vijayan J, Sharma VK (2014) Cerebral Venous Thrombosis: A Path Less Travelled and making a Difference. Brain Disord Ther 3: e112. doi:10.4172/2168-975X.1000e112

Page 2 of 2

results of the International Study on Cerebral Vein and Dural Sinus Thrombosis (ISCVT). Stroke 35: 664-670.

7. Saposnik G, Barinagarrementeria F, Brown RD Jr, Bushell CD, Cucchiara B et al. (2011) American Heart Association Stroke Council and the Council on Epidemiology and Prevention. Diagnosis and management of cerebral venous thrombosis: a statement for healthcare professionals from the American Heart Association/American Stroke Association. Stroke 42: 1158-1192.

8. Lewis MB, Bousser MG (1999) Cerebral venous thrombosis: nothing, heparin, or local thrombolysis? Stroke 30: 1729.
9. Stam J, Majoie CB, van Delden OM, van Lienden KP, Reekers JA (2008) Endovascular thrombectomy and thrombolysis for severe cerebral sinus thrombosis: a prospective study. Stroke 39: 1487-1490.

10. Coutinho JM, Majoie CB, Coert BA, Stam J (2009) Decompressive hemicraniectomy in cerebral sinus thrombosis: consecutive case series and review of the literature. Stroke 40: 2233-2235.

11. Coutinho JM, Majoie CB, Coert BA, Stam J (2009) Decompressive hemicraniectomy in cerebral sinus thrombosis: consecutive case series and review of the literature. Stroke 40: 2233-2235. 